\title{
$\begin{array}{ll}\text { Research Square } & \text { Preprints are preliminary reports that have not undergone peer review. } \\ \text { They should not be considered conclusive, used to inform clinical practice, } \\ \text { or referenced by the media as validated information }\end{array}$
}

\section{The prognostic values and clinical implications of m6A methylation regulators in epithelial ovarian cancer}

huimin shen ( $\nabla$ shenhm@126.com)

The first affiliated hospital, sun yat-sen university https://orcid.org/0000-0002-5758-9202

Tiefeng Cao

the first affiliated hospital, sun yat-sen university

Jinhui Li

the first affiliated hospital, sun yat-sen university

\section{Research article}

Keywords: RNA modification, methyltransferase, demethylases, epigenetics, prognostic signature

Posted Date: March 13th, 2020

DOI: https://doi.org/10.21203/rs.3.rs-17066/v1

License: () (i) This work is licensed under a Creative Commons Attribution 4.0 International License. Read Full License 


\section{Abstract}

Background: N6-methyladenosine (m6A) exists in both DNA and RNA modification. RNA m6A modification drives tumor initiation and metastasis through regulating cancer stem cells. However, the detailed mechanisms and the distinct m6A regulatory gene type underlying ovarian cancer mRNA modification remain unclear.

Results: We analyzed copy number variation (CNVs) and mRNA expression of ovarian cancer cases in TCGA dataset to determine the copy number variation patterns of m6A regulatory genes, and the associations between m6A dysregulation or certain regulatory gene and overall survival. We showed the KIAA1429, as the writer gene, had highest amplification percentage and were associated with overall survival, or disease-free survival, whereas the associations with prognostic survival were independent of other prognostic factors including stage, grade, and debulking status of the tumour. Besides, METTL14 and YTHDC2, one as the writer gene and the other as reader gene, was also related with clinical outcome. Furthermore, subgroups analysis addressed that m6A upregulation especially writer gain contributed to prognosis in epithelial ovarian cancer.

Conclusions: Collectively, our data addressed that m6A upregulation is likely to be critical to the clinical outcome, and KIAA1429 showed the highest correlation with clinical outcome in ovarian cancer among m6A regulatory genes.

\section{Background}

Epithelial ovarian cancer (EOC) is the most prevalent cause of death in gynecologic malignancies. The patients are usually diagnosed at advanced stages for the lacking of clinically meaningful biomarkers for early screening and understanding of molecular pathogenesis. Debulking surgery followed by chemotherapy can cause 50-80\% complete clinical response in advanced stage cases. However, most cases will relapse and eventually die for recurrence or metastasis[1, 2]. Recent studies focused on target therapy or immune therapy which is proven to prolong survival time in advanced-stage ovarian cancer patients. But the molecular pathogenesis and mechanism is underestimated. Hence, finding the functionally relevant molecular biomarkers and new therapeutic targets are still challenging.

N6-methyladenosine (m6A) exists in both DNA and RNA modification, namely m6dA and m6A. Recently, m6A is proved to be the most abundant in mRNA modification, and the most powerful in the regulation of mRNA[3]. M6A regulators consists of many catalysase including methyltransferase "writers" (WTAP, METTL3, METTL14, MT-A70 and KIAA1429), binding proteins "readers" (YTHDF1, YTHDF2, YTHDF3, YTHDC1 and YTHDC2), and demethylase "erasers" (FTO and ALKBH5)[4].

M6A dysregulation exists in various process including stem cell fates[5], somatic cell reprogramming[6], and causes impaired cell proliferation, self-renewal capacity, developmental defects and cell death[7]. Articles showed that RNA m6A modification drives tumor initiation and metastasis through regulating cancer stem cells[8]. It is found to be associated with tumorigenesis in different cancers types, including breast cancer[9], hematologic malignancies[10, 11]. Specially, the overall level of $\mathrm{m} 6 \mathrm{~A}$ regulators is found to be higher in ovaries compared to other organs and tissues[12], suggesting that m6A modification plays a critical role in reproduction system. One finding suggested that METTL3 has an oncogenic role in ovarian cancer development and aggressiveness [13]. The other finding showed that ALKBH5 was a candidate oncogene and a potential target for ovarian cancer therapy[14]. However, due to the heterogeneity and bias, comprehensive analysis is needed to analysis the expression of $\mathrm{m} 6 \mathrm{~A}$ regulators, the roles of m6A regulators in cancer progression and metastasis, the function in clinical survival, the correlation between m6A regulators and clinicopathological characteristics in ovarian cancer. Hence, we systematically analyzed the ovarian cancer cases from the TCGA database including the clinical and sequencing data of 579 cases, evaluated the expression spectrum of specific m6A gene, and addressed the association between the genetic alteration or mRNA expression and clinicopathological features or clinical outcomes. In this study, we found that the alteration and mRNA expression level of m6A RNA methylation regulators play a critical role in EOC progression and metastasis.

\section{Results}

\section{Alteration of m6A regulatory genes in EOC patients}

With the analysis from sequencing data in TCGA, only 20 independent samples were identified with m6A genes mutations (Table 1). TP53 was shown with high mutation (88\%) in this cohort, which is in line with published literatures[15]. However, copy number variations (CNVs) of m6A regulatory genes that included deep deletion/shallow deletion/ copy number gain/ amplification, had high frequency in EOC cases (Fig. 1A), with loss of copy number (3584/5733) in most of the CNV events (Fig. 1B, Table 2). Moreover, for alteration only including amplification and deep deletion as shown in cBioportal database, m6 A regulatory genes showed higher frequency of amplification (Fig. 1C, Fig S1A). Among them, KIAA1429 or VIRMA (writer) showed the highest frequency of amplification (85/576) in this cohort (Fig. 1D, Fig S1B), WTAP (14/576) and YTHDC2 (14/576) had relative high frequency of deep deletion (Fig. 1E, Table 2), which are $\mathrm{m} 6 \mathrm{~A}$ "writer" and "reader" genes, showing the important of writers in m6A regulation. 
Table 1

Mutation of $\mathrm{m6A}$ regulatory genes in epithelial ovarian cancer patients from TCGA dataset.

\begin{tabular}{|c|c|c|c|c|c|c|c|c|c|c|c|c|c|}
\hline $\begin{array}{l}\text { Sample } \\
\text { ID }\end{array}$ & ALKBH5 & FTO & METTL14 & METTL3 & WTAP & VIRMA & RBM15B & RBM15 & ZC3H13 & YTHDF1 & YTHDF2 & YTHDF3 & YTHDC1 \\
\hline $\begin{array}{l}\text { TCGA- } \\
10- \\
0938\end{array}$ & & & & & & & & & & H365Y & & & \\
\hline $\begin{array}{l}\text { TCGA- } \\
13- \\
0920\end{array}$ & & S246I & & & & & & & & V200I & & & \\
\hline $\begin{array}{l}\text { TCGA- } \\
23- \\
1117\end{array}$ & & & & & & & & & & & R355W & & \\
\hline $\begin{array}{l}\text { TCGA- } \\
25- \\
1319\end{array}$ & & & & & & & & & & & & & R575* \\
\hline $\begin{array}{l}\text { TCGA- } \\
61- \\
2008\end{array}$ & & & & & & & & G563A & & & & & \\
\hline $\begin{array}{l}\text { TCGA- } \\
09- \\
2051\end{array}$ & & & & & & & & & K385N & & & & \\
\hline $\begin{array}{l}\text { TCGA- } \\
24- \\
2271\end{array}$ & Q174R & & & & & & & & & & & & \\
\hline
\end{tabular}

Table 2

CNV patterns in epithelial ovarian cancer samples $(n=576)$

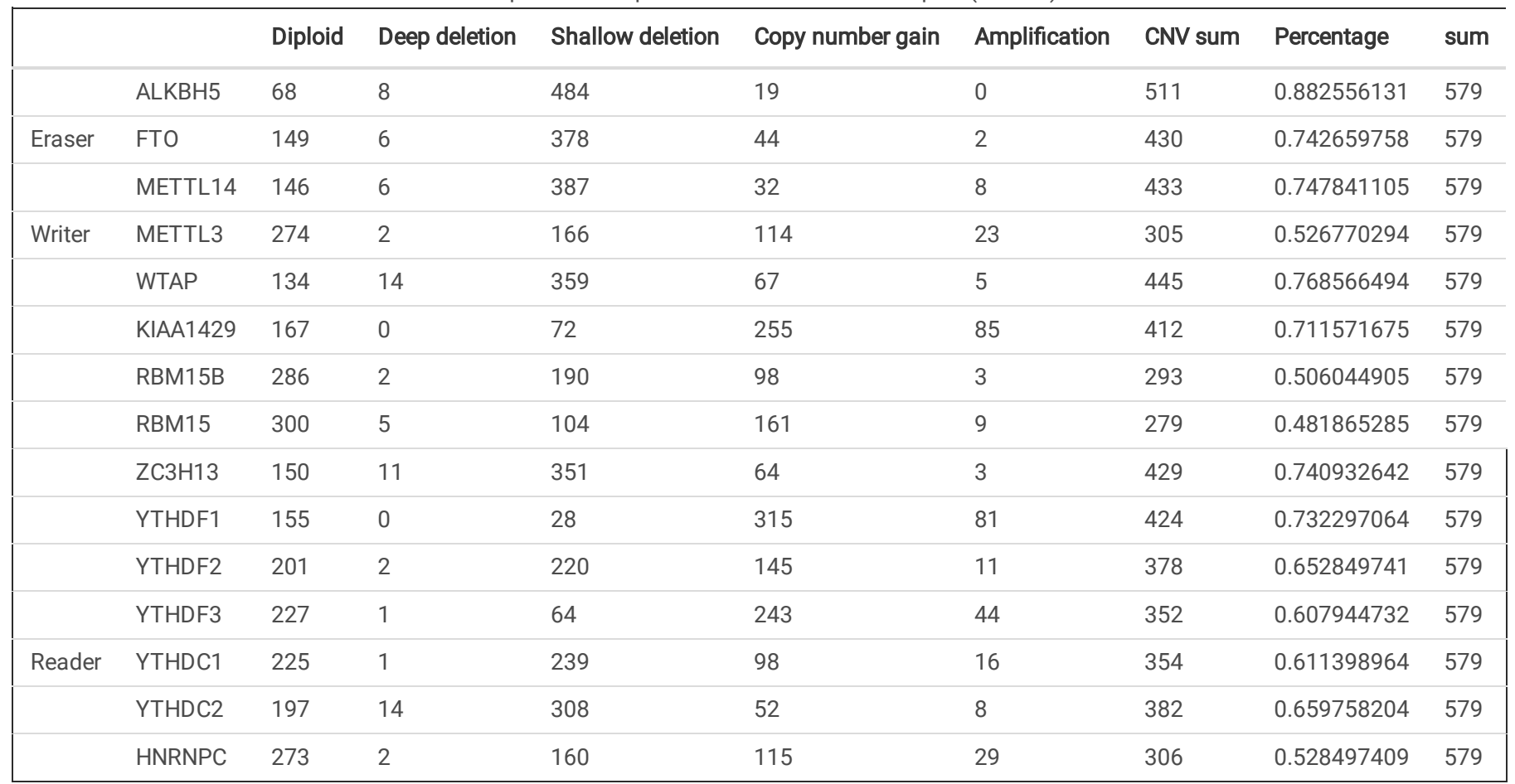

\section{Correlation between M6A regulatory genes alterations and clinicopathological factors}

To address the correlation between alteration (deep deletion or amplification) of m6A regulatory genes and the clinicopathological and molecular characteristics, we assessed the related prognostic factors including age, stage, grade, debulking status, and chemotherapy sensitivity. The results depicts that the amplification or deep deletion of m6A regulatory genes were associated with grade (Table $3, p=0.016$ ), but not with age, stage, debulking status and chemotherapy response. It is reported that missense mutations of TP53 were found to be the most frequent, while mutations of BRCA1 and BRCA2 showed the critical prognostic value in ovarian cancers[16]. TP53 and BRCA mutation play a critical role in the progression and pathogenesis of ovarian cancer. Here we evaluated the association between the variation of m6A regulatory genes and the mutation of these genes. In fact, the alterations of m6A regulatory genes were not significantly correlated with BRCA and TP53 mutations (Table S1), which indicated that m6A regulation affects ovarian cancer through other critical genes and molecular pathways. 
Table 3

Clinicopathological features of EOC patients with or without m6A regulatory genes' alteration. Alteration, amplification or deep deletion.

\begin{tabular}{|c|c|c|c|c|}
\hline & & With alteration & Without alteration & $\mathbf{p}$ \\
\hline \multirow[t]{2}{*}{ age } & $<=60$ & 139 & 175 & 0.479 \\
\hline & $>60$ & 120 & 134 & \\
\hline \multirow[t]{4}{*}{ Stage } & I & 6 & 11 & 0.213 \\
\hline & II & 11 & 19 & \\
\hline & III & 191 & 242 & \\
\hline & IV & 47 & 40 & \\
\hline \multirow[t]{3}{*}{ Grade } & G1 & 0 & 6 & 0.016 \\
\hline & $\mathrm{G} 2$ & 24 & 43 & \\
\hline & G3 & 227 & 252 & \\
\hline \multirow[t]{2}{*}{ Debulking status } & Optimal & 161 & 201 & 0.495 \\
\hline & Sub-optimal & 67 & 73 & \\
\hline \multirow[t]{2}{*}{ Chemotherapy sensitivity } & Sensitivity & 88 & 106 & 0.259 \\
\hline & Resistant & 34 & 55 & \\
\hline
\end{tabular}

\section{Association between CNVs of m6A regulatory genes and survival of EOC patients}

To identify the association between m6A regulatory genes alteration and ovarian cancer patients' survival, we evaluated the clinical value of CNVs on the overall survival (OS) and progression-free survival (PFS) among all ovarian cancer patients. Figure 2A-2B addressed that there was no significant difference of OS or PFS between individuals with or without alteration of m6A regulatory genes including gene amplification or deep deletion. Because cases number was limited and small with amplification or deep deletion in individual regulatory gene, here we analyzed the prognostic value of alteration with the CNVs data in individual gene. We found that copy number gain or amplification of KIAA1429 (writer, Hazard Ratio [HR] $=0.70, p=0.0012$ ), while shallow deletion or deep deletion of YTHDC2 (reader, HR = 0.75, p = 0.0087) were related with improved OS and PFS, and copy number gain of METTL14 (writer) was bordline significantly related with poor OS but not PFS (Fig. 2C \& 2D for OS and PFS, respectively). The prognostic value of other ten genes was shown in Fig S2 with no significant difference between different subgroups based on CNVs. The results also showed that writer genes, a group of methyltransferase enzymes, might be important for patient survival.

Next, we conducted survival analysis with subgroups of CNVs (copy number gain of writer genes and deletion of eraser genes) to test the prognostic role of $\mathrm{m} 6 \mathrm{~A}$ regulatory genes especially the writers. Figure 3A\&3B depicts that in eraser deletion patients, the group combination with writer genes gain had worse OS and DFS than those without writer genes gain. This evidence supported the correlation between up-regulated m6A level (especially writer genes gain) and poor survival. By univariable Cox regression analysis adjusted for prognostic factors including stage, grade, BRCA mutation, age, debulking status, and subgroups,

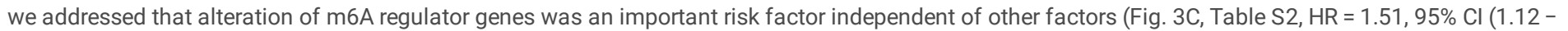
$2.1), p=0.008)$. Furthermore, KIAA1429 was independent risk factor for overall survival when conducting multivariate analysis adjusted for prognostic factors (Fig. 3D, Table S3, HR = 1.30, 95\% Cl (1.13-1.51), p<0.001).

\section{Association between m6A genes level and survival in EOC patients}

The effects of alterations in m6A regulatory genes on the mRNA expression were evaluated. Figure $4 \mathrm{~A}-4 \mathrm{C}$ addressed that the mRNA expression were significantly correlated with the diverse CNV patterns. Higher mRNA level was shown to be related with amplification or copy number gains for all the m6A regulator genes, while decreased mRNA expression was shown in shallow deletion or deep deletions for the m6A regulator genes.

Considering the important role of m6A regulator genes in pathological feature and survival above with the CNV data, we investigated the relationship between individual m6A regulator genes mRNA level and clinical survival. K-M analysis was conducted with the expression data in the TCGA dataset. Figure 5A and Fig. 5B depicts the unadjusted HRs and $95 \%$ confidence intervals for medium of gene expression levels with OS and PFS. 376 patients with restriction to samples with mRNA data were included for analysis. KIAA1429 (HR=1.27, $p=0.063$, Fig. 5C), YTHDC2 (HR = 1.31, p = 0.039, Fig. 5D) were most significantly associated with poor overall survival, and YTHDC2 ( $\mathrm{HR}=1.39, \mathrm{p}=0.011$, Fig. 5E) showed significant correlation with poor PFS. However, KIAA1429 showed no significant association with the poor PFS but with the tendency (Fig. 5F). In general, the results were in consistent with the prognostic value of m6A genes alteration above that copy number gain or amplification of writers was associated with poor prognosis, illustrating that high m6A expression correlated the poorer outcome.

\section{Validation of prognostic role of KIAA1429 in EOC patients}

To validate the prognostic significance of KIAA1429 expression in ovarian cancer patients, we integrated the expression profiling in Kaplan-Meier plotter online database (K-M plotter). We evaluated the prognostic value of KIAA1429 at mRNA level by Kaplan-Meier plotter analysis with cases enrolled from multiple GEO (Gene Expression Omnibus) datasets. Figure 6A \& 6B showed that higher KIAA1429 expression was correlated with shorter overall survival by detecting with 
different probes (223110_at, HR = $\left.1.36(1.1-1.67), p=0.0038 \& 238818 \_a t, H R=1.31(1.06-1.64), p=0.014\right)$, with the PFS shown in Fig. 6C \& 6D (HR = 1.31 $(1.08-1.6), p=0.0055 ; H R=1.37(1.13-1.66), p=0.0015$, respectively).

\section{Gene set enrichment analysis of KIAA1429 expression level}

As mentioned above, m6A upregulation, especially KIAA1429 gain, is important to promote cancer pathogenesis and progression. Herein we evaluated the molecular mechanism of m6A upregulation in EOC by GSEA analysis (Gene set enrichment analysis). We explored the gene set enrichment between samples with different KIAA1429 mRNA expression. The GSEA results showed that high KIAA1429 expression was associated with some critical pathway. Nuclear hormone receptor binding, small conjugating protein ligase activity, acid amino acid ligase activity, positive regulation of transcription from RNA polymerase II promoter, and positive regulation of RNA metabolic process were related with KIAA1429 upregulation in EOC patients, as Table 4 and Fig. 7A-E shown. However, the further mechanism and regulation are needed to be illustrated.

Table 4

Gene sets enrichment with subgroups of KIAA1429 expression level in quartile.

\begin{tabular}{|lccccc|}
\hline GS DETAILS & SIZE & ES & NES & NOM p-val & FDR q-val \\
\hline NUCLEAR_HORMONE_RECEPTOR_BINDING & 28 & 0.67 & 2.13 & 0 & 0.038 \\
\hline SMALL_CONJUGATING_PROTEIN_LIGASE_ACTIVITY & 50 & 0.57 & 2.12 & 0 & 0.019 \\
\hline ACID_AMINO_ACID_LIGASE_ACTIVITY & 56 & 0.56 & 2.12 & 0 & 0.013 \\
\hline TRANSCRIPTION_FROM_RNA_POLYMERASE_II_PROMOTER & 64 & 0.58 & 2.11 & 0 & 0.011 \\
\hline RNA_METABOLIC_PROCESS & 118 & 0.53 & 2.01 & 0 & 0.020 \\
\hline
\end{tabular}

\section{Discussion}

N6-methyladenosine ( $\mathrm{m6A}$ ) plays important roles in cancer progression and metastasis by controlling cell differentiation and cell pluripotency especially cancer stem cells[17]. It has been shown that m6A modification affect the breast cancer stem cells enrichment through changing the NANOG mRNA stability, relating with a shorter survival of breast cancer cases[18, 19]. In human lung cancer, METTL3 can promote oncogene translation by decreasing epidermal growth factor receptor (EGFR) protein expression[20]. Besides, m6A regulatory genes also show an critical oncogenic role in acute myeloid leukemia[21], glioblastoma[8, 22], and hepatocellular carcinoma[18].

Especially, Xiaoyao Lin et al. revealed that m6A modification of mRNAs regulates epithelial-mesenchymal transition (EMT) which is an important process for cancer cell metastasis[23]. Upregulation of METTL3, one of the writers in m6A regulatory genes, was proved to be shown in ovarian carcinoma, and significantly related with some prognostic clinicopathological factors including grade, lymph-node metastasis, and stage. Interestingly, METTL3 can promote EMT by upregulating the receptor tyrosine kinase AXL to promote ovarian cancer progression[13]. Moreover, other m6A modification genes, such as m6 demethylases FTO and ALKBH5, can regulate the Wnt/ $\beta$-catenin pathway, contributing to PARP inhibitor resistance in BRCA-mutated EOC cells[24]. The published data showed that $\mathrm{m} 6 \mathrm{~A}$ regulation play an important role in ovarian cancer progression. In this article, we integratively analyzed the CNVs and mRNA expression data from m6A regulator genes in TCGA dataset. We explored the prognostic value of distinct writers, readers, and erasers, the association of copy number alteration and prognosis, and the specific gene regulator for the ovarian cancer prognosis.

Surprisely, we found that KIAA1429, METTL14 and YTHDC2 were associated with cliniopathological features and clinical outcomes. Firstly, we addressed that amplification of KIAA1429 which showed highest among the thirteen genes' amplification, and METTL14 had correlation with clinicopathological grade and poor prognosis, which is consistent with the data shown in previous published studies. Besides, we elucidated the KIAA1429 mRNA expression and its potential clinical significance in epithelial ovarian cancer. The results depicted that KIAA1429 upregulation was related with poor prognosis, which is in accordance with the relationship between CNV and clinical prognosis. This was proved in breast cancer that KIAA1429 was highly expressed in breast cancer tissues and down-regulated in non-cancerous breast tissues. The prognosis, especially overall survival of breast cancer was associated with KIAA1429 expression. METTL14, one of the writers in m6A regulatory genes and one important component of the methyltransferase complex, plays a critical oncogenic role in human AMLs and liver cancer by positioning RNA substrates for methylation medicated by a complex comprising RBM15-WTAP-METTL3METTL14[8, 18, 25-27]. Further- more, our findings also found that amplification of YTHDC2, as an m6A modification reader, was correlated with poor clinical outcome in ovarian cancer, which is shown in studies that YTHDC2 promotes cancer metastasis[28]

We identified the mechanism of KIAA1429 mRNA expression on clinical prognosis. Finally, 19624 genes were enrolled into the GSEA process. We addressed that positive regulation of transcription from RNA polymerase II promoter and positive regulation of RNA metabolic process were critical pathways, while articles showed that N6-methyladenosine promote cancer progression through regulating RNA metabolism[17]. As shown by the subgroups analysis, writers gain plus eraser loss showed association with poor prognosis in EOC. As methyltransferase enzymes, writer genes play an important role and the most part in $\mathrm{m} 6 \mathrm{~A}$ regulation process. The results implied that the regulation of $\mathrm{m} 6 \mathrm{~A}$ level might be associated with writers' expression and function.

Our articles showed high ALKBH5 loss in EOC but lower alteration including deep deletion and amplification. It is ambiguous about the role of ALKBH5 or the m6A regulatory genes loss in ovarian cancer. ALKBH5 can induce the stem cell phenotype in breast by mediating the m6A-demethylation of NANOG mRNA[19], and further studies proved that ALKBH5 regulated the malignant behavior of glioblastoma[29]. In ovarian cancer, ALKBH5 may be a potential oncogene in EOC by inhibiting autophagy of epithelial ovarian cancer through regulating miR-7 and BCL-2[14]. Our article showed no relationship between ALKBH5 CNVs or mRNA expression level with prognostic value including overall survival and prognostic free survival, which is addressed with TCGA database 
and validated with K-M plotter database. The reason for the inconsistent may be for the limited clinical samples and cell lines which can be solved. In the current study, we used a vast spectrum of tumour samples from the publicity online studies and datasets. This indicated that our conclusions are general.

But there were still some shortcomings in this article. We used the CNVs and expression datasets from TCGA dataset in which the sample size may be limited. Then we used GEO as the validation set, which includes a large sample size to improve repeatability. This approach may increase statistical power, but limitation exists insofar as some patients' information will be lost and data integrity will be impaired. Also, GEO samples have the possibility that patients are likely to have differences in clinical characteristics, and treatment. These differences affected the association between the m6A regulator genes and clinical outcome, with the possibility that we increased the false negative ratio.

In summary, this study addressed the critical role of m6A upregulation, especially writers' gain or amplification or mRNA upregulation, in clinical outcome of ovarian cancer, providing the prognostic role and possible targets for tumor diagnosis and prognosis. Analysis with large-scale genomic data from databases can reveal the role of m6A methylation regulator genes that is an important part of RNA stability, methylation and functions.

\section{Material And Methods Data collection}

We download the CNV data, mRNA expression, mutation, and clinical data of TCGA dataset by cBioportal platform (https://www.cbioportal.org/) and TCGAassembler (http://www.compgenome.org/TCGA-Assembler/), which is open for the public.

\section{Data processing}

There are 579 ovarian cancer patients with CNV data from the TCGA database. Segmentation analysis and GISTIC algorithm were used to identify the copynumber alteration[30]. There are 316 samples with mutation data and CNVs information. 379 cases were included with mRNA expression data and 3 replicates were removed by patient id. Clinical data and prognostic factors included survival data (overall survival and progression free survival), age, stage, grade, debulking status (optimal and suboptimal), and chemotherapy response (sensitivity and resistant).

\section{Gene set enrichment analysis (GSEA)}

Gene set enrichment analysis (http://software.broadinstitute.org/- gsea/index.jsp) is a method to detect the critical biological process in ovarian cancer by genes enrichment analysis comparing two distinct biological status. In our study, two groups were included according the quartile of KIAA1429 mRNA expression level, with one group are cases with lowest quartile expression and the other group with highest quartile expression. Process with normalized $\mathrm{p}$ value $<0.05$ and FDR $<0.25$ were identified to be significantly enriched.

\section{Kaplan-Meier (K-M) plotter database}

The Kaplan Meier plotter (http://kmplot.com/analysis) is based on an online database[31] and is capable to assess the association of genes on survival in ovarian cancer. K-M plotter was mainly used in validation for the prognosis value of KIAA1429 mRNA expression. The correlation of individual KIAA1429 mRNA expression and survival in ovarian cancer was analyzed online and presented with the hazard ratio, 95\% confidence intervals and computed log rank pvalue.

\section{Statistical analysis}

All the statistical analysis was conducted by R language 3.6.1 (https://www.r- project.org/). We analyzed the association between clinicopathological features or prognostic factors with the CNV or mRNA expression level of m6A regulatory genes with chi-square test. Kaplan-Meier curve and Cox regression analysis were used for univariate or multivariate analysis and for evaluation of the prognosis value of m6A regulatory gene. Cox proportional hazard regression model was performed using $\mathrm{R}$ language. Results with a $\mathrm{p}$-value $<0.05$ were considered to be significant.

\section{Abbreviations}

m6A: methylation of N6 adenosine; EOC: epithelial ovarian cancer; TCGA: the Cancer Genome Atlas; CNV: copy number variation; OS: overall survival; DFS: disease-free survival; PFS: progression-free survival; HR: Hazard ratio; K-M plotter: Kaplan-Meier plotter; GEO: Gene Expression Omnibus; EMT: epithelialmesenchymal transition; mRNA: messenger RNA; GIST: Genomic Identification of Significant Targets in Cancer; AML: acute myelocytic leukemia; ROS: reactive oxygen species; HIF-a: hypoxia induced factor-a; GSEA: gene set enrichment analysis

\section{Declarations}

Ethical approval and consent to participate: Not applicable. This article does not contain any studies with human participants or animals performed by any of the authors.

Consent for publication: Not applicable.

Availability of data and material[The datasets generated and analysed during the current study are available from TCGA, GEO, and Kaplan-Meier plotter that provide free online tools and resources

Competing interest: The authors declare that they have no competing interests. 
Funding: Not applicable.

Authors' contributions: CT and JL searched the database and analysis data. CT wrote the main manuscript text and prepared the table. SH critically reviewed the manuscript. All authors read and approved the final manuscript.

Acknowledgments: We are grateful for the contribution of all databases including TCGA, GEO, and Kaplan-Meier plotter that provide free online tools and resources.

\section{References}

1. Hennessy BT, Coleman RL and Markman M. Ovarian cancer. Lancet. 2009; 374(9698):1371-1382.

2. Rojas V, Hirshfield KM, Ganesan S and Rodriguez-Rodriguez L. Molecular Characterization of Epithelial Ovarian Cancer: Implications for Diagnosis and Treatment. Int J Mol Sci. 2016; 17(12).

3. Yue Y, Liu J and He C. RNA N6-methyladenosine methylation in post-transcriptional gene expression regulation. Genes Dev. 2015; 29(13):1343-1355.

4. Ji P, Wang X, Xie N and Li Y. N6-Methyladenosine in RNA and DNA: An Epitranscriptomic and Epigenetic Player Implicated in Determination of Stem Cell Fate. Stem Cells Int. 2018; 2018:3256524.

5. Wang Y, Li Y, Toth JI, Petroski MD, Zhang Z and Zhao JC. N6-methyladenosine modification destabilizes developmental regulators in embryonic stem cells. Nat Cell Biol. 2014; 16(2):191-198.

6. Chen T, Hao YJ, Zhang Y, Li MM, Wang M, Han W, Wu Y, Lv Y, Hao J, Wang L, Li A, Yang Y, Jin KX, et al. m(6)A RNA methylation is regulated by microRNAs and promotes reprogramming to pluripotency. Cell Stem Cell. 2015; 16(3):289-301.

7. Liu N and Pan T. N6-methyladenosine-encoded epitranscriptomics. Nature structural \& molecular biology. 2016; 23(2):98-102.

8. Cui Q, Shi H, Ye P, Li L, Qu Q, Sun G, Sun G, Lu Z, Huang Y, Yang CG, Riggs AD, He C and Shi Y. m(6)A RNA Methylation Regulates the Self-Renewal and Tumorigenesis of Glioblastoma Stem Cells. Cell Rep. 2017; 18(11):2622-2634.

9. Niu Y, Lin Z, Wan A, Chen H, Liang H, Sun L, Wang Y, Li X, Xiong XF, Wei B, Wu X and Wan G. RNA N6-methyladenosine demethylase FTO promotes breast tumor progression through inhibiting BNIP3. Molecular cancer. 2019; 18(1):46.

10. Bansal H, Yihua Q, lyer SP, Ganapathy S, Proia DA, Penalva LO, Uren PJ, Suresh U, Carew JS, Karnad AB, Weitman S, Tomlinson GE, Rao MK, et al. WTAP is a novel oncogenic protein in acute myeloid leukemia. Leukemia. 2014; 28(5):1171-1174.

11. Vu LP, Pickering BF, Cheng Y, Zaccara S, Nguyen D, Minuesa G, Chou T, Chow A, Saletore Y, MacKay M, Schulman J, Famulare C, Patel M, et al. The N(6)methyladenosine (m(6)A)-forming enzyme METTL3 controls myeloid differentiation of normal hematopoietic and leukemia cells. Nat Med. 2017; 23(11):1369-1376.

12. Lence T, Akhtar J, Bayer M, Schmid K, Spindler L, Ho CH, Kreim N, Andrade-Navarro MA, Poeck B, Helm M and Roignant JY. m(6)A modulates neuronal functions and sex determination in Drosophila. Nature. 2016; 540(7632):242-247.

13. Hua W, Zhao Y, Jin X, Yu D, He J, Xie D and Duan P. METTL3 promotes ovarian carcinoma growth and invasion through the regulation of AXL translation and epithelial to mesenchymal transition. Gynecol Oncol. 2018; 151(2):356-365.

14. Zhu H, Gan X, Jiang X, Diao S, Wu H and Hu J. ALKBH5 inhibited autophagy of epithelial ovarian cancer through miR-7 and BCL-2. Journal of experimental \& clinical cancer research : CR. 2019; 38(1):163.

15. Iwanicki MP, Chen HY, lavarone C, Zervantonakis IK, Muranen T, Novak M, Ince TA, Drapkin R and Brugge JS. Mutant p53 regulates ovarian cancer transformed phenotypes through autocrine matrix deposition. JCl Insight. 2016; 1(10).

16. Ramus SJ and Gayther SA. The contribution of BRCA1 and BRCA2 to ovarian cancer. Mol Oncol. 2009; 3(2):138-150.

17. Dai D, Wang H, Zhu L, Jin H and Wang X. N6-methyladenosine links RNA metabolism to cancer progression. Cell Death Dis. $2018 ; 9(2): 124$.

18. Ma JZ, Yang F, Zhou CC, Liu F, Yuan JH, Wang F, Wang TT, Xu QG, Zhou WP and Sun SH. METTL14 suppresses the metastatic potential of hepatocellular carcinoma by modulating N(6) -methyladenosine-dependent primary MicroRNA processing. Hepatology (Baltimore, Md). 2017; 65(2):529-543.

19. Zhang C, Samanta D, Lu H, Bullen JW, Zhang H, Chen I, He X and Semenza GL. Hypoxia induces the breast cancer stem cell phenotype by HIF-dependent and ALKBH5-mediated m(6)A-demethylation of NANOG mRNA. Proceedings of the National Academy of Sciences of the United States of America. 2016; 113(14):E2047-2056.

20. Lin S, Choe J, Du P, Triboulet R and Gregory RI. The m(6)A Methyltransferase METTL3 Promotes Translation in Human Cancer Cells. Molecular cell. 2016; 62(3):335-345

21. Wang J, Muntean AG and Hess JL. ECSASB2 mediates MLL degradation during hematopoietic differentiation. Blood. 2012; 119(5):1151-1161.

22. Yang Z, Li J, Feng G, Gao S, Wang Y, Zhang S, Liu Y, Ye L, Li Y and Zhang X. MicroRNA-145 Modulates N(6)-Methyladenosine Levels by Targeting the 3'Untranslated mRNA Region of the N(6)-Methyladenosine Binding YTH Domain Family 2 Protein. J Biol Chem. 2017; $292(9): 3614-3623$.

23. Lin X, Chai G, Wu Y, Li J, Chen F, Liu J, Luo G, Tauler J, Du J, Lin S, He C and Wang H. RNA m(6)A methylation regulates the epithelial mesenchymal transition of cancer cells and translation of Snail. Nat Commun. 2019; 10(1):2065.

24. Fukumoto T, Zhu H, Nacarelli T, Karakashev S, Fatkhutdinov N, Wu S, Liu P, Kossenkov AV, Showe LC, Jean S, Zhang L and Zhang R. N(6)-Methylation of Adenosine of FZD10 mRNA Contributes to PARP Inhibitor Resistance. Cancer research. 2019; 79(11):2812-2820.

25. Weng H, Huang H, Wu H, Qin X, Zhao BS, Dong L, Shi H, Skibbe J, Shen C, Hu C, Sheng Y, Wang Y, Wunderlich M, et al. METTL14 Inhibits Hematopoietic Stem/Progenitor Differentiation and Promotes Leukemogenesis via mRNA m(6)A Modification. Cell Stem Cell. 2018; 22(2):191-205 e199.

26. Jaffrey SR and Kharas MG. Emerging links between m(6)A and misregulated mRNA methylation in cancer. Genome Med. 2017; 9(1):2. 
27. Patil DP, Chen CK, Pickering BF, Chow A, Jackson C, Guttman M and Jaffrey SR. m(6)A RNA methylation promotes XIST-mediated transcriptional repression. Nature. 2016; 537(7620):369-373.

28. Tanabe A, Tanikawa K, Tsunetomi M, Takai K, Ikeda H, Konno J, Torigoe T, Maeda H, Kutomi G, Okita K, Mori M and Sahara H. RNA helicase YTHDC2 promotes cancer metastasis via the enhancement of the efficiency by which HIF-1alpha mRNA is translated. Cancer letters. 2016; 376(1):34-42.

29. Zhang S, Zhao BS, Zhou A, Lin K, Zheng S, Lu Z, Chen Y, Sulman EP, Xie K, Bogler O, Majumder S, He C and Huang S. m(6)A Demethylase ALKBH5 Maintains Tumorigenicity of Glioblastoma Stem-like Cells by Sustaining FOXM1 Expression and Cell Proliferation Program. Cancer cell. 2017; 31(4):591606.e596.

30. Mermel CH, Schumacher SE, Hill B, Meyerson ML, Beroukhim R and Getz G. GISTIC2.0 facilitates sensitive and confident localization of the targets of focal somatic copy-number alteration in human cancers. Genome biology. 2011; 12(4):R41.

31. Gyorffy B, Lanczky A, Eklund AC, Denkert C, Budczies J, Li Q and Szallasi Z. An online survival analysis tool to rapidly assess the effect of 22,277 genes on breast cancer prognosis using microarray data of 1,809 patients. Breast cancer research and treatment. 2010; 123(3):725-731.

\section{Figures}

A

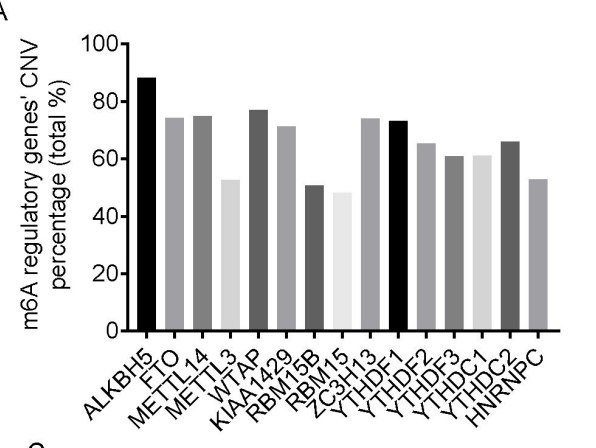

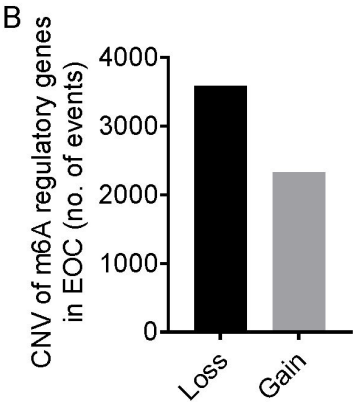
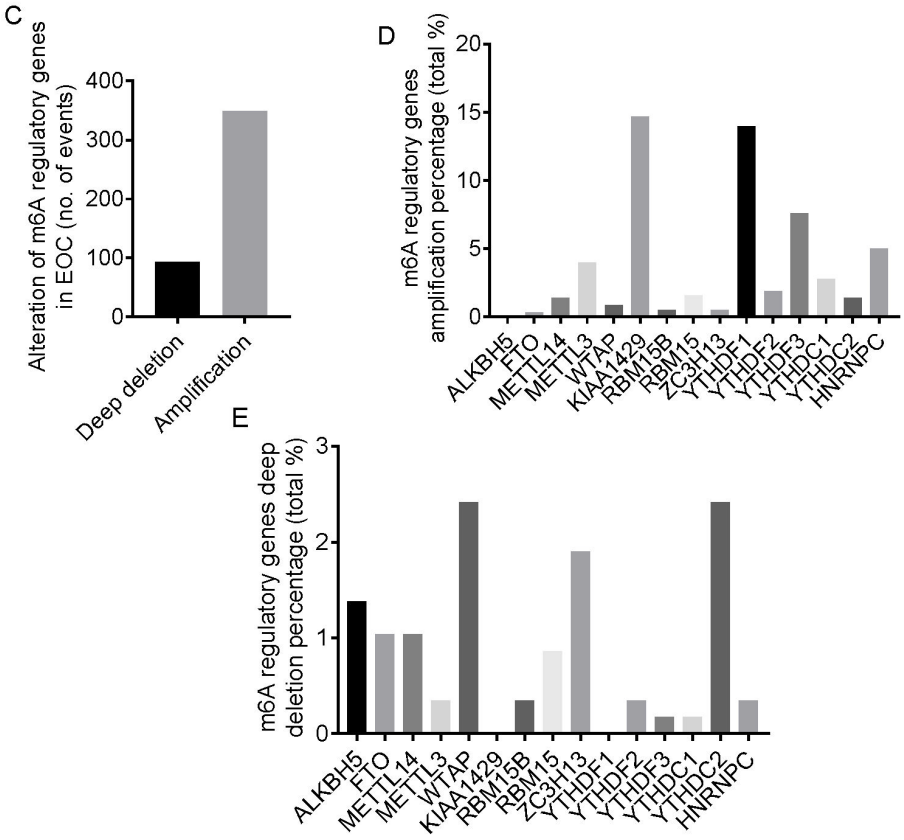

Figure 1

Alteration of m6A regulatory genes in EOC patients 
A

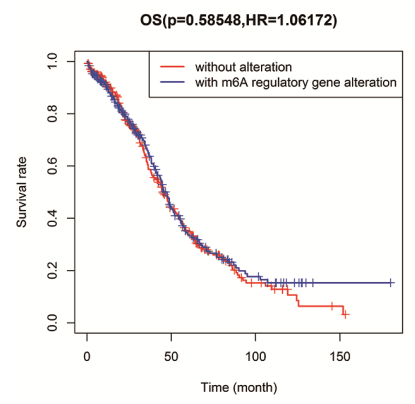

D

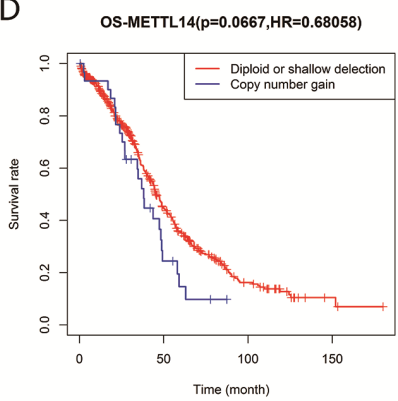

G

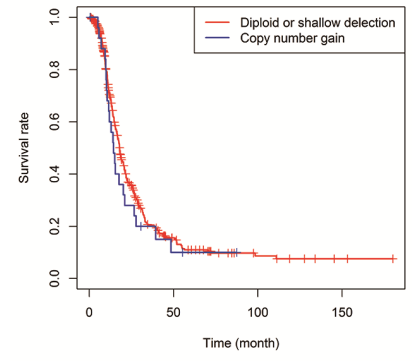

B

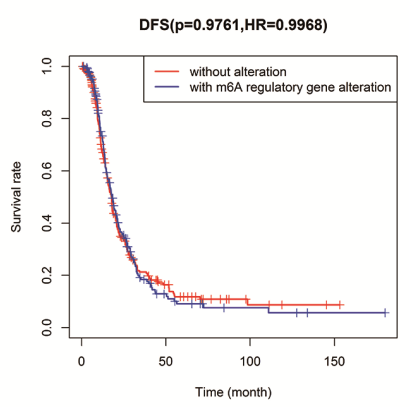

E

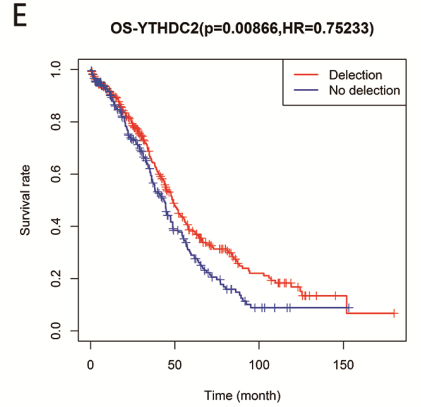

$\mathrm{H} \quad$ DFS-YTHDC2(p=0.05336,HR $=0.81364)$

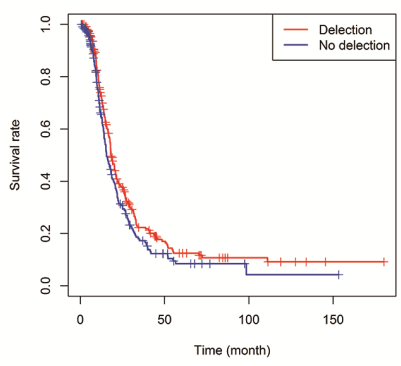

C

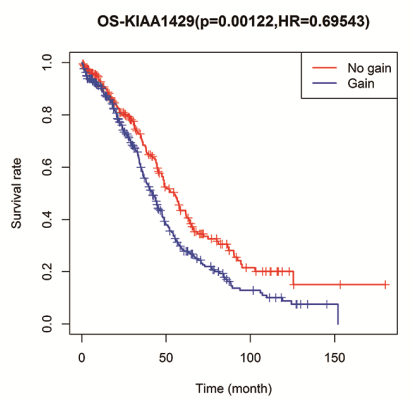

F DFS-KIAA1429(p=0.01518,HR=0.76861)

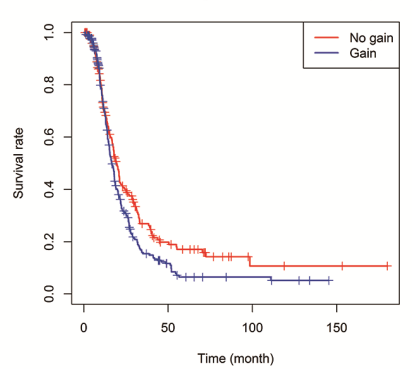

\section{Figure 2}

Clinical value of CNVs on the overall survival (OS) and progression-free survival (PFS) among all ovarian cancer patients. 
A
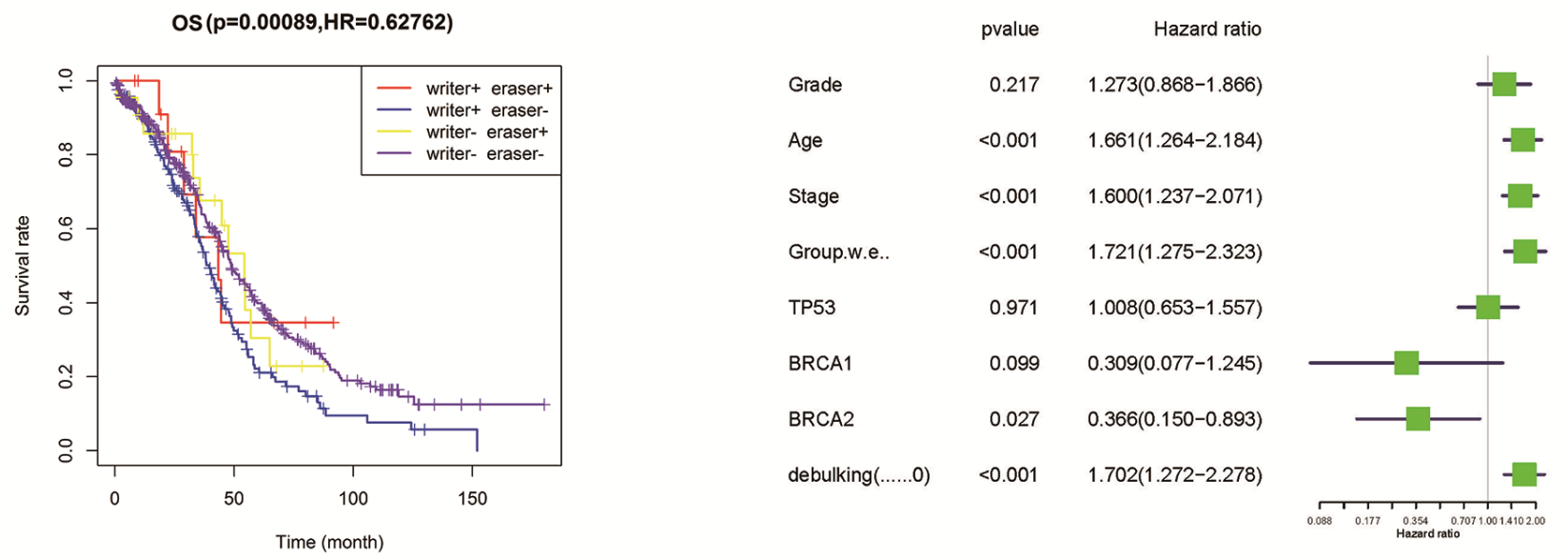

B

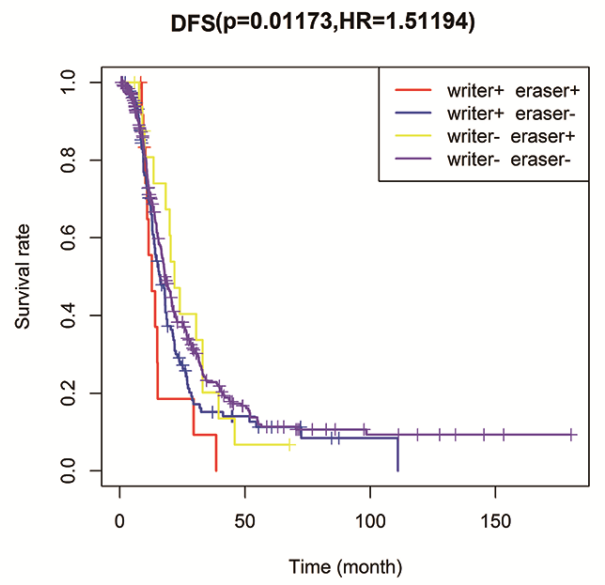

D

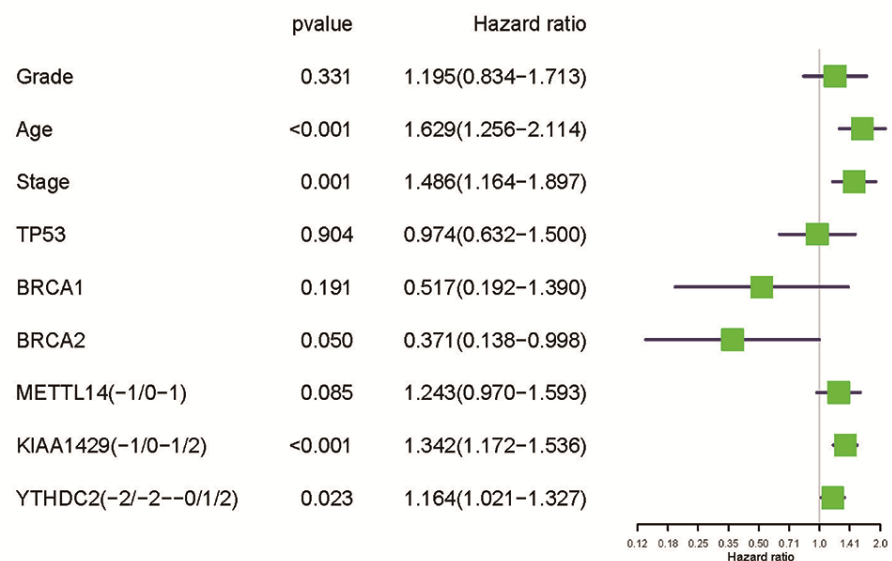

\section{Figure 3}

Survival analysis with subgroups of CNVs

A

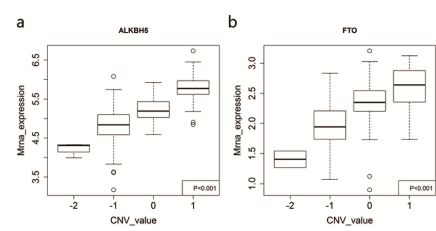

B
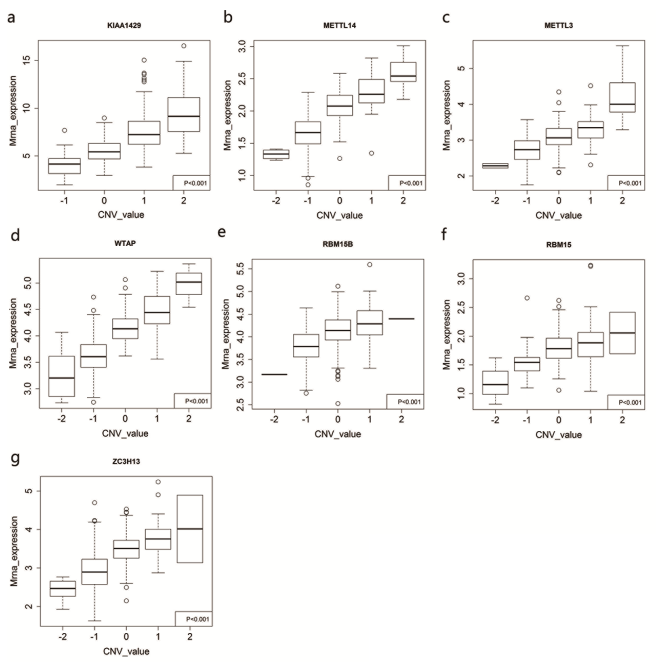
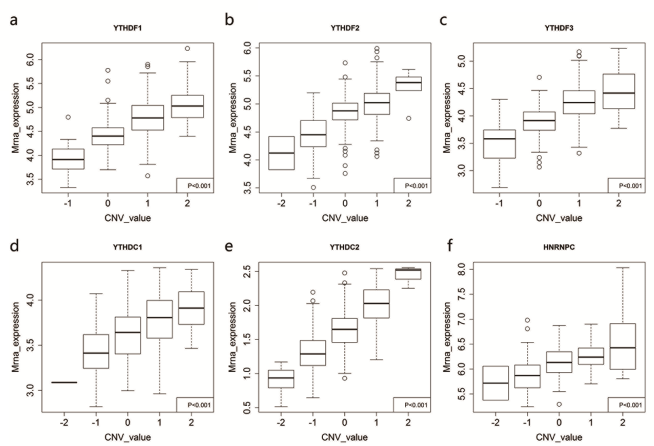

Figure 4

Evaluation of the effects of alterations in m6A regulatory genes on the mRNA expression 
A

$\begin{array}{lrr}\text { Gene } & \text { pvalue } & \text { Hazard ratio } \\ \text { YTHDC2 } & 0.039 & 1.307(1.009-1.694) \\ \text { KIAA1429 } & 0.063 & 1.272(1.002-1.650) \\ \text { METTL3 } & 0.116 & 0.814(0.628-1.056) \\ \text { YTHDF3 } & 0.146 & 1.209(0.932-1.568) \\ \text { YTHDC1 } & 0.209 & 0.849(0.655-1.101) \\ \text { RBM15B } & 0.322 & 0.879(0.679-1.138) \\ \text { WTAP } & 0.369 & 1.125(0.868-1.457) \\ \text { YTHDF1 } & 0.437 & 1.107(0.854-1.434) \\ \text { FTO } & 0.439 & 1.106(0.854-1.432) \\ \text { METTL14 } & 0.458 & 1.102(0.851-1.427) \\ \text { RBM15 } & 0.741 & 0.958(0.740-1.240) \\ \text { ALKBH5 } & 0.762 & 0.961(0.742-1.245) \\ \text { YTHDF2 } & 0.841 & 0.974(0.752-1.261) \\ \text { HNRNPC } & 0.854 & 0.976(0.754-1.264) \\ \text { ZC3H13 } & 0.922 & 1.013(0.782-1.312)\end{array}$

C

OS- KIAA1429( $\mathrm{p}=0.06343, \mathrm{HR}=1.27236)$

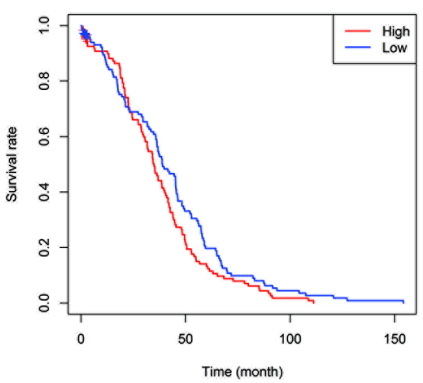

F

DFS-KIAA1429( $\mathrm{p}=0.16286, \mathrm{HR}=1.19925)$

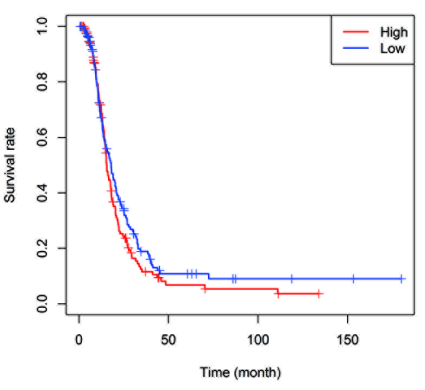

B
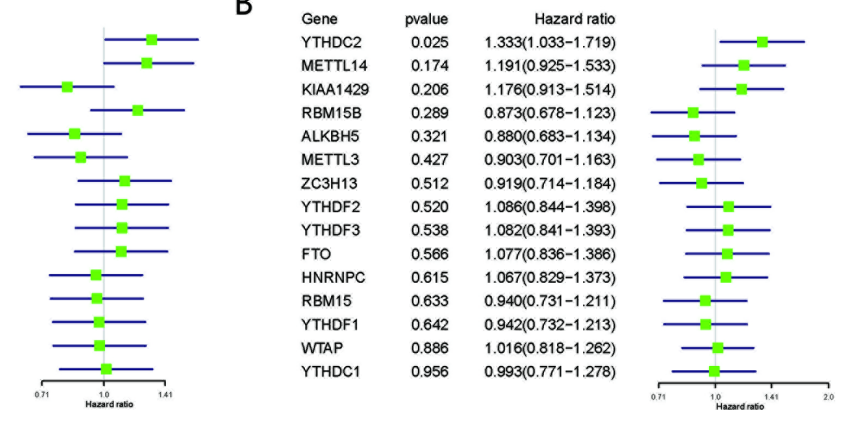

E
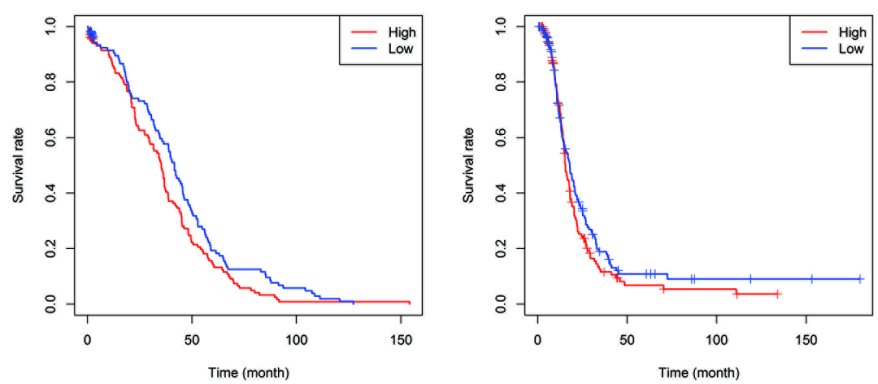

\section{Figure 5}

K-M analysis was conducted with the expression data in the TCGA dataset. 
A

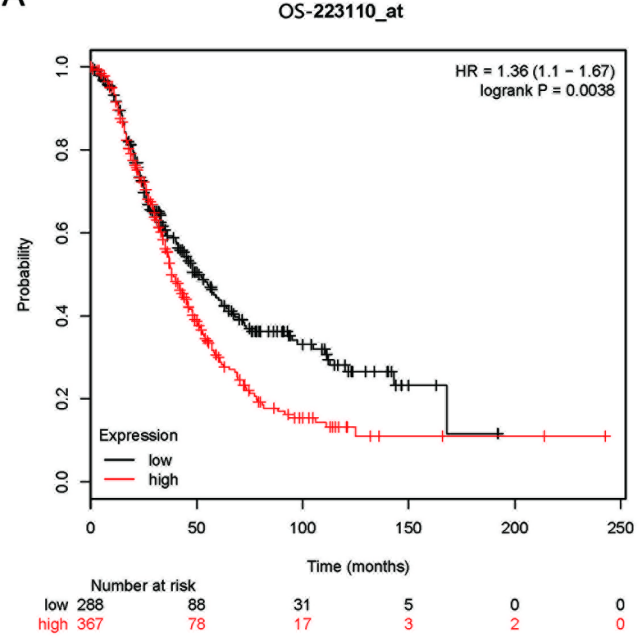

C

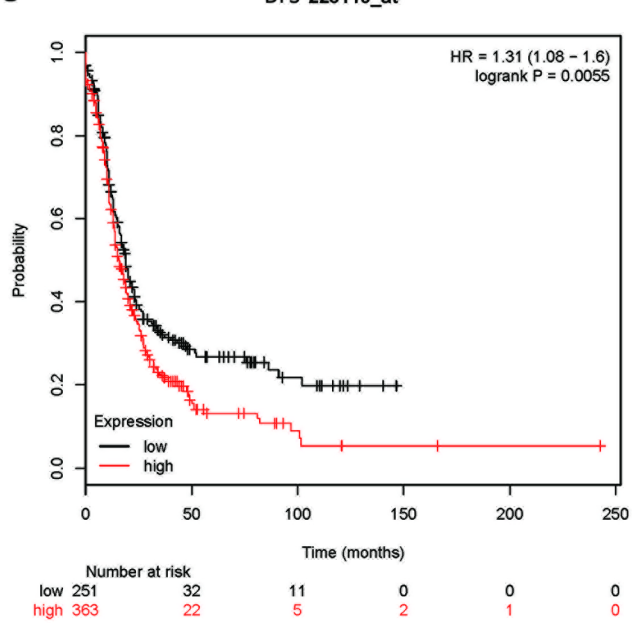

B

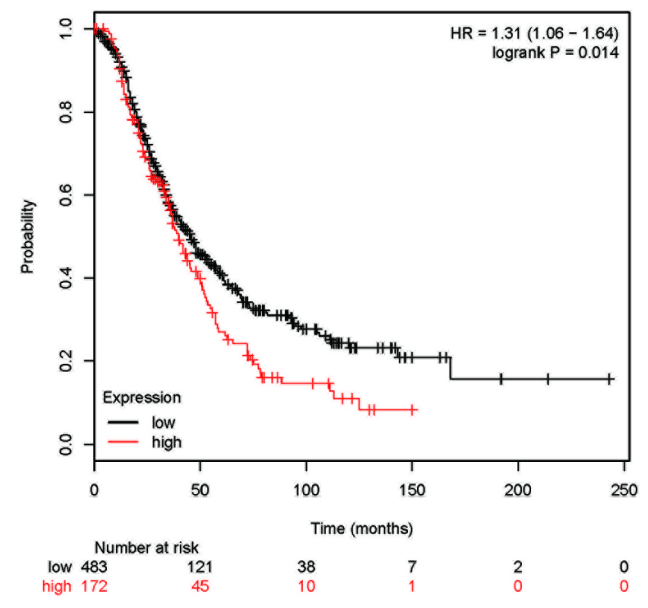

D

DFS-238818_at

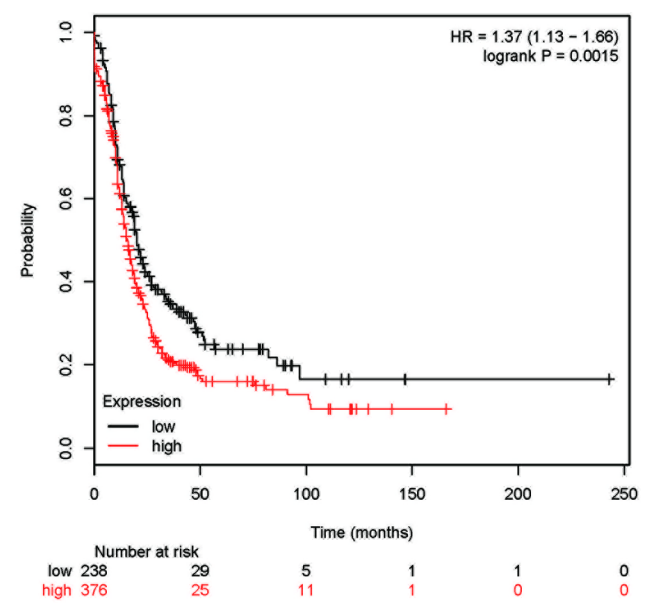

Figure 6

The prognostic value of KIAA1429 at mRNA level by Kaplan-Meier plotter analysis with cases enrolled from multiple GEO (Gene Expression Omnibus) datasets. 


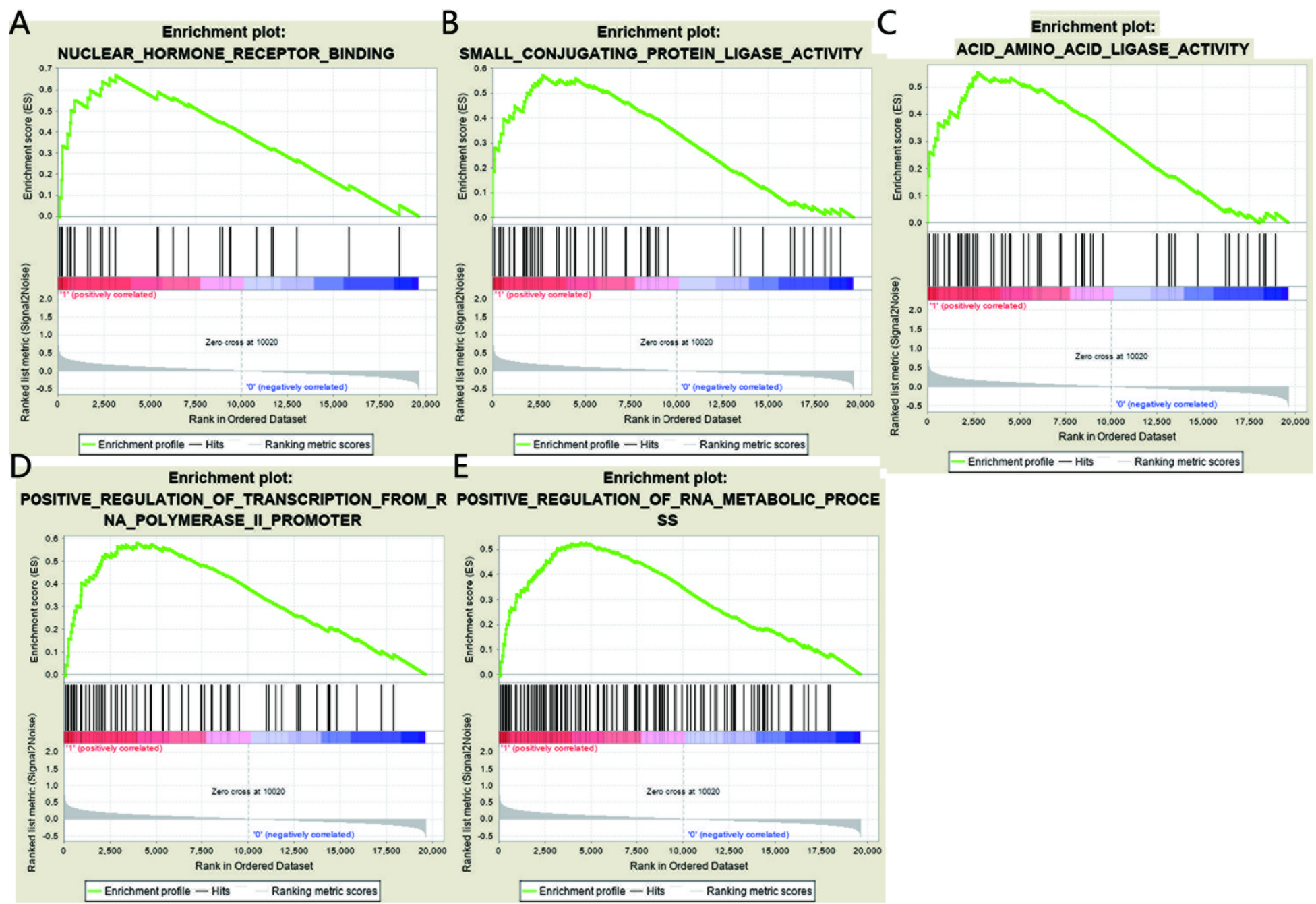

Figure 7

Nuclear hormone receptor binding, small conjugating protein ligase activity, acid amino acid ligase activity, positive regulation of transcription from RNA polymerase II promoter, and positive regulation of RNA metabolic process were related with KIAA1429 upregulation in EOC patients

\section{Supplementary Files}

This is a list of supplementary files associated with this preprint. Click to download.

- Tables1.docx

- tableS3.docx

- Figs1.tiff

- figs2.tiff

- tables2.docx 